\title{
Willkommen IM DSChUNGEL - GLAM, HARdCORE und Metal in Los Angeles
}

\section{Dietmar Elflein}

Die Geschichte populärer Musik kann auch als ein Netzwerk regionaler oder lokaler Szenen und der mit ihnen verbundenen Musiken gelesen werden. Historisch wirkungsmächtige Stile populärer Musik lassen sich so als Weiterentwicklungen ehemals regionaler Stile deuten, die - aus welchen Gründen und auf welche Art auch immer - internationale Beachtung gefunden haben. Im Extremfall wird der Name einer Stadt mit einer bestimmten Epoche oder Stilistik populärer Musik (z.B. Chicago Blues) gleichgesetzt. Regional betrachtet bilden meist Institutionen wie Auftrittsorte, Tonstudios und Schallplattenfirmen wichtige Knotenpunkte in solch einem Netzwerk, um die sich dann Musikerinnen und Musiker scharen können, die ähnliche musikalische Interessen verfolgen oder eine - wie auch immer begrenzte öffentliche Wahrnehmung ihrer Musik suchen (vgl. Slobin 2000: 11-23). Das Verhältnis solch regionaler Szenen und ihrer jeweiligen Sounds zu ihrer geographischen, sozialen und kulturellen Umgebung, wie z.B. der Stadt, ist komplex und besteht aus sich widersprechenden und ergänzenden Diskursen. Im Spannungsfeld von Punk und Hard Rock bzw. Heavy Metal entwickelten sich zwischen 1975 und 1985 im Großraum Los Angeles parallel mehrere unterschiedliche Modelle solch regionaler Musiken und Zentren:

- die Glam-, Hair Metal- und Sleaze-Szene rund um die Auftrittsorte am Sunset Strip,

- die Heavy Metal-Szene rund um die Schallplattenfirma Metal Blade Records und

- die Hardcore-Szene rund um die Band Black Flag mit ihrer Schallplattenfirma SST Records.

Jede dieser Musiken und Szenen ist ein Netzwerkknoten im Netzwerk der Geschichte populärer Musik - keine ist jedoch zum klanglichen Synonym für Los Angeles geworden. Sie können aber stellvertretend für Organisations- 
formen regionaler Szenen stehen, in denen sich Stilistiken populärer Musik zentrieren.

Zwischen den drei ausgewählten regionalen Szenen kam es immer wieder zu Berührungspunkten, Austausch und auch Rivalitäten. Im Sinne einer methodischen Trennschärfe wird im Folgenden vor allem das Trennende, die Unterschiedlichkeit betont, um die verschiedenen Organisationsmuster herausarbeiten zu können. Zu Beginn soll jedoch der gemeinsame regionale Ursprung, Los Angeles und das, was darunter in diesem speziellen Fall zu verstehen ist, etwas genauer definiert werden.

\section{Mythos Los Angeles}

Los Angeles erscheint oft weniger als ein konkreter, geographisch bestimmbarer Ort im Süden Kaliforniens als vielmehr ein populär-kultureller Mythos, der sich aus unendlich vielen Büchern, Filmen und Musikstücken speist. Mike Davis (1994) hat diesen in seinem Buch City of Quartz ausführlich beschrieben und auch entmythologisiert. Das Bild, das gerade außerhalb der USA von dem konkreten Ort Los Angeles existiert, entspricht selten den Tatsachen. Los Angeles ist zudem in seiner Geschichte und Struktur nicht oder nur begrenzt vergleichbar mit deutschen oder europäischen Metropolen, Großstädten und Ballungsräumen und die Dynamik seiner Entwicklung ist auch nur bedingt auf andere US-amerikanische Ballungsräume übertragbar.

Als Los Angeles wird häufig - und so auch in diesem Artikel - die so genannte Greater L.A. Area bezeichnet, die kein politisches Gebilde ist und weder mit der City of Los Angeles noch mit dem County Los Angeles übereinstimmt. Die geographischen Zentren der drei hier in Frage stehenden regionalen Szenen lassen sich zum Teil im Los Angeles County, zum Teil in der City of Los Angeles und zum Teil auch in Orange County lokalisieren. So liegt der Sunset Strip - Zentrum der Glam- und Hair Metal-Szene - im Los Angeles County. Die Hardcore-Szene entwickelte sich vor allem in den strandnahen Vorstädten, die entweder zum County oder zur City gehören und verbreitete sich schnell auch nach Orange County und damit in die Greater L.A. Area. Die Metal-Szene rund um das Label Metal Blade besteht aus Musikern und Fans aus dem Los Angeles County und dem Orange County. Der Sitz von Metal Blade Records liegt in Sherman Oaks, dem einzigen hier besprochenen Gebiet, das ausschließlich zur City of Los Angeles gehört.

Die Greater L.A. Area wird statistisch definiert als die aus Los Angeles County und Orange County bestehende Los Angeles-Long Beach-Santa Ana Metropolitan Area mit ca. 12 Millionen Einwohnern auf einer Fläche von 
$12.562 \mathrm{~km}^{2}$. Die Bevölkerungsdichte ist mit 1.029 Einwohnern $/ \mathrm{km}^{2}$ vergleichsweise gering. ${ }^{1}$ Ein Grund hierfür ist der hohe Anteil an Einfamilienhäusern an der Gesamtzahl der zur Verfügung stehenden Wohneinheiten, der mit 45,5\% (City of Los Angeles), 56,2\% (Los Angeles County) bzw. 62,2\% Prozent (Orange County) erheblich größer ist als z.B. in der BRD mit 28,4\% Prozent. ${ }^{2}$ Im County von Los Angeles leben knapp zehn Millionen, in der City of Los Angeles 3,7 Millionen Menschen.

Eigenständige Städte innerhalb dieser Metropolitan Area darf man sich nun nicht als im europäischen Sinne geschlossene Siedlungsräume vorstellen, die jeweils von Gebieten umgeben sind, die zum County gehören. Vielmehr existiert ein unübersichtlicher Flickenteppich von Nachbarschaften und Siedlungsgebieten mit wechselnden regionalen Zugehörigkeiten. Beispielsweise ist West-Hollywood, zu dem der Sunset Strip gehört, eine eigenständige, zum County Los Angeles gehörende Stadt, die jedoch komplett von der City of Los Angeles umgeben ist.

Diese von Davis (1994: 179-256) ausführlich beschriebene Ausgründung von Siedlungsgebieten aus der City of Los Angeles erfolgte wegen der Möglichkeit einer eigenständigen Steuergesetzgebung sowie eines eigenen Flächennutzungsplans und zielte auf eine Wertsteigerung der Einfamilienhäuser über eine Regulierung des Zuzugs weiterer Bewohner nach ökonomischen und auch rassischen Kriterien. In West Hollywood leben beispielsweise 36.000 Menschen, deren Zusammensetzung sich deutlich vom Durchschnitt von County und City of Los Angeles unterscheidet. Während die Hispanics im County mit 47,2 Prozent die größte Bevölkerungsgruppe stellen - gefolgt von Weißen (29\%), Asiaten (12,8\%) und Afroamerikanern $(8,6 \%)$, sind in West

1 Zum Vergleich: Berlin 3.810 Einwohner $/ \mathrm{km}^{2}$, Hamburg 2.312, London 4.700 und New York City 10.359. Quelle für die metropolitan und combined statistical areas ist das U.S. Census Bureau (http://www.census.gov/population/www/ estimates/Estimates\%20pages_final.html; Stand vom 22.11.2006).

2 Zahlen für die Greater L.A. Area liegen nicht vor. Gleiches gilt für Berlin und andere europäische Großstädte. In Hamburg liegt der Anteil der Ein- und Zweifamilienhäuser am Gesamtwohnungsbestand bei 20,7\%. Für die USA insgesamt liegt der Anteil der Einfamilienhäuser am Gesamtwohnungsbestand bei 66,8\%. Die Zahlen für die USA entstammen dem 2005 American Community Survey (http://factfinder.census.gov/home/saff/main.html?_lang=en; Stand vom 22.11.2006). Die Zahlen für die BRD und Hamburg entsprechen den Angaben des Statistischen Bundesamtes bzw. des Statistikamtes Nord (http://www-ec.destatis.de/csp/shop/sfg/bpm.html.cms.cBroker.cls?cmspath= struktur,vollanzeige.csp\&ID=1019434 bzw. http://www.statistik-nord.de/index. php?id=457; Stand vom 22.11.2006). 
Hollywood nur 9\% der Bevölkerung Hispanics, dagegen aber mindestens 75\% der Bevölkerung Weiße. ${ }^{3}$

\section{Glam, Hair Metal und Sleaze - der Sunset Strip}

Der in West Hollywood gelegene Teil des Sunset Boulevard wird auf einer Länge von ca. zwei Kilometern als Sunset Strip bezeichnet und ist seit den Anfängen der Filmindustrie ein Zentrum des Nachtlebens in der Greater L.A. Area, da er in räumlicher Nähe zu den großen Filmstudios und einigen Zentralen der internationalen Unterhaltungsindustrie liegt. Dementsprechend bilden Auftrittsorte und Personen, die einen - vermeintlichen oder realen - Zugang zur Unterhaltungsindustrie versprechen, die Knotenpunkte, um die sich Szenen der populären Musik entwickeln. Musikerinnen und Musiker reisen aus den ganzen USA zum Sunset Strip, alle mit dem Traum, in Klubs wie dem Whisky, dem Troubadour oder dem House of Blues den ökonomischen Durchbruch zu schaffen. In der Folge prägten bereits seit den 1960er Jahren jugendliche Ausreißer und auch jugendliche Obdachlose die Szenen am Sunset Strip entscheidend mit.

Wie Penelope Spheeris in ihrem Dokumentarfilm von 1988 The Decline of the Western Civilisation Part II: The Metal Years zeigt, galt dies auch für die Glam- und Hair Metal-Szene am Sunset Strip. Die von ihr interviewte Band London - in den frühen 1980er Jahren immerhin der erste Karriereschritt am Sunset Strip für spätere Popstars wie Blackie Lawless (W.A.S.P.), Nikki Sixx (Mötley Crüe), Slash und Izzy Stradlin (beide Guns N' Roses) - ist zum Zeitpunkt des Interviews obdachlos und lebt gemeinsam in einem Wohnmobil. Das Video zu »Welcome To The Jungle«, dem ersten Hit von Guns N' Roses (1987), thematisiert den gleichen Zusammenhang: Es inszeniert die Ankunft des Sängers Axl Rose am Sunset Strip per Bus, während Gitarrist Slash vermeintlich obdachlos und trinkend auf dem Bürgersteig sitzt. Slash ist übrigens das einzige Mitglied von Guns N' Roses, das in Hollywood bzw. Los Angeles aufgewachsen ist. Viele der mit der Glam-, Hair Metal- und Sleaze-Szene verbundenen Musiker sind der Anziehungskraft des Sunset Strip erlegen und zugezogen - Risiken wie mögliche Obdachlosigkeit wurden und werden in Kauf genommen. Der oben erwähnte Blackie Lawless stammt aus Staten Island, New York, Nikki Sixx aus San José, Kalifornien.

3 Die entsprechenden Zahlen für die City of Los Angeles differieren nur leicht von den für das County genannten (http://factfinder.census.gov/home/ saff/main. html?_lang=en; Stand vom 22.11.2006). 
Die Glam Metal-Band Poison, um ein letztes Beispiel anzuführen, zog gemeinsam aus Pennsylvania nach Los Angeles und vervollständigte hier ihre Besetzung mit einem aus New York zugezogenen Gitarristen. Rikki Rockett, der Schlagzeuger von Poison, beschreibt im Interview mit Spheeris den Alltag eines Musikers am Sunset Strip wie folgt:

»P. Spheeris: `So there was Poison, multimillion dollar band out there on the street handing out flyers? Ricky Rockett: >This is how we did it actually. During the day we would rehearse and at night we'd go to clubs and hand stuff out, you know, we'd try to look all cool and stuff [...], putting all our flyers up the wall, coming up another band and they cover up and then you'd sneak back. It's a war! « (Spheeris 1988).

Werbung für die eigene Band erscheint damit neben dem eigentlichen Musizieren als zentrale Aktivität. Im folgenden Interviewzitat aus derselben Dokumentation deutet sich darüber hinaus eine ökonomische Überlebensstrategie der Musiker an:

»Shea Darek [Schlagzeuger der Band London]: >I never had a job, the girls have always been very generous.< [...] Rikki Rockett: >In a sense it's a form of prostitution « (ebd.).

Man lebte auf Kosten von Frauen, die man an den Abenden in oder vor den Klubs kennenlernte und denen man sich als kommender Star präsentierte. Die Werbeflugblätter für die eigene Band dienten dabei als Beweis des Musikerdaseins.

Zwei weitere zentrale Punkte lassen sich aus diesen Interviewzitaten aus Spheeris' Dokumentarfilm destillieren: Die Szene erscheint erstens weniger als Gemeinschaft, denn als Ort der Konkurrenz, in dem jeder vor allem um das eigene Wohl besorgt ist. Bands wurden gegründet und bei (ökonomisch) besseren Angeboten ohne Skrupel wieder verlassen - die oben angeführten London sind hierfür ein gutes Beispiel. Die Band hat von ihren berühmt gewordenen Ex-Mitgliedern nie wirklich profitieren können. Zweitens ist es zentral, sowohl als Individuum als auch als Band visuell aufzufallen, wie es z.B. Rikki Rockett von Poison beschreibt. Musikalische Fähigkeiten alleine reichen nicht automatisch aus - weder um Mitglied einer Band zu werden, noch um als Band einen Auftritt zu ergattern und ein Publikum sowie möglicherweise Vertreter von Plattenfirmen zu interessieren.

Image und (Bühnen-)Show sind in der Glam-, Hair Metal- und SleazeSzene deshalb sehr wichtig. Insofern ist es auch nicht verwunderlich, dass sich die Stile hinter den drei genannten Begriffen weniger musikalisch als vielmehr visuell abgrenzen lassen. Glam entspricht dabei einem vordergründig androgynen Image, dessen auffälligste Elemente die Verwendung 
von Make-up, toupierte und wahlweise blond oder schwarz gefärbte lange Haare sowie aus dem Bereich der Fetischerotik entlehnte Lederbekleidung sind. Der Begriff Hair Metal bezieht sich vor allem auf die verbreiteten toupierten und gefärbten Langhaarfrisuren. Dabei ist die Betonung androgyner Elemente in Glam und Hair Metal laut Robert Walser weniger als beginnende Auflösung von Geschlechterstereotypen, denn als Hypermaskulinität zu verstehen. Walser zitiert in Dunn/McFaydens DVD Metal - A Headbanger's Journey (2006) aus dem Leserbrief eines Fans, in dem dieser von »having the guts to be glam « spricht und folgert daraus: »Being feminine is the most masculine thing you can do «. Sleaze ist dagegen eher an klischeehafte modische Vorstellungen über Motorradfahrer angelehnt und betont Tätowierungen, um Stirn, Arm oder Bein gebundene Tücher, zerrissene Jeans und schwarzes Leder - ein Image, das bereits im US-Hard Rock der 1970er Jahre präsent ist, z.B. bei Aerosmith. Glam, Hair Metal und Sleaze modernisierten ihr Image über die Hereinnahme von modischen Elementen aus dem Punk und dem britischen Glam der 1970er Jahre und passten es so unter anderem den visuellen Bedürfnissen des Musikvideosenders MTV an. MTV ging in den USA 1981 auf Sendung und spielte ab 1983 verstärkt Bands aus der hier behandelten Szene, während visuell eher unscheinbare US-amerikanische Hard Rock-Bands der 1970er Jahre wie REO Speedwagon in der kommerziellen Bedeutungslosigkeit verschwanden (vgl. Christie 2003: 153-171). Bands wie beispielsweise Guns N' Roses wechselten im Laufe ihrer Karriere zwischen den hier angedeuteten visuellen Images, ohne dass dies einen Einfluss auf die von ihnen gespielte Musik hatte.

Aerosmith waren nicht nur modische Vorbilder für Teile der Szene, sie waren auch ein starker musikalischer Einfluss. ${ }^{4}$ Gespielt wurde meist in einer Besetzung mit zwei Gitarren, Bassgitarre, Schlagzeug und Gesang. Es erklingt ein eindeutig Blues basierter Hard Rock mit klarer Aufteilung in Solo und Rhythmusgitarre, einem durchgehenden Puls der Bassgitarre und Backbeat basiertem Schlagzeugspiel. Die Songstrukturen basieren meist auf einem »Intro, two verses, break, verse, playout«-Schema, das laut Allan F. Moore (2001: 150) typisch für Rockmusik seit der Beat-Ära ist. Gitarrenriffs sind zwar häufig die tragenden Elemente des Songs, basieren jedoch meist noch auf aus dem Blues entlehnten Harmoniefolgen.

Wichtig - gerade bezogen auf MTV und die Möglichkeit auch im USamerikanischen Formatradio gespielt zu werden - sind zudem so genannte Powerballaden, deren hervorstechendes Element meist auf akustischen

4 Gerade Guns N' Roses sind ohne Aerosmith musikalisch kaum denkbar. Man vergleiche bei Interesse die beiden Alben Rocks (Aerosmith 1976) und Appetite for Destruction (Guns N' Roses 1987). 
Gitarren gezupfte Akkordbrechungen sind, die im Refrain mit dezenten Powerchords und wahlweise auch Streichern verstärkt werden. Mehr vom Glam der 1970er Jahre beeinflusste Bands wie Mötley Crüe, die bereits erwähnten Poison oder auch Quiet Riot spielen dagegen meist in einer Besetzung mit nur einer E-Gitarre. Quiet Riot landeten 1983 mit einer Coverversion der britischen Glam-Band Slade, »Cum On Feel The Noize«, den bis dato größten Hit der Glam Metal-Szene am Sunset Strip. Das zugehörige Album Metal Health (1983) schaffte es im selben Jahr sogar bis auf Platz eins der Billboard Charts.

1977 gründete sich in Hollywood das Musicians Institute mit seinen vielfältigen Unterabteilungen wie Guitar Institute (GIT), Bass Institute (BIT) etc. und brachte ab Ende der 1970er Jahre Absolventen z.B. in »advanced metal guitar « hervor. Diese drängten ebenfalls in die Klubs und Bands am Sunset Strip und bereicherten die Szene um handwerklich hervorragend ausgebildete Instrumentalisten, die ihre Virtuosität auch gerne demonstrierten. Der Glam und/oder Blues basierte Hard Rock am Sunset Strip wurde somit auch durch Soli modernisiert, die als Showeinlagen dienten. Als Vorreiter können Van Halen gelten, deren Gitarrensolo »Eruption« von 1978 Robert Walser (1993: 67-78) bereits ausführlich betrachtet hat.

In der medialen Wahrnehmung wurde die Szene am Sunset Strip - und mit ihr auch die weitere Modernisierung des US-Hard Rock - Anfang der 1990er Jahre von Nirvana und der so genannten Grunge-Bewegung abgelöst. In kommerzieller Hinsicht ist diese Abfolge nicht so eindeutig. So verkauften Guns N' Roses von ihrem 1987 erschienen Debüt in den USA laut Angabe der Recording Industry Association of America (RIAA) 14 Millionen Einheiten, während Nirvana von ihrem 1991 erschienen Album Nevermind nur 10 Millionen verkauften. Metallicas so genanntes »schwarzes Album « aus demselben Jahr ging in den USA 14 Millionen Mal über die Ladentische. ${ }^{5}$

5 Quelle für alle Zahlen: http://www.riaa.com/gp/bestsellers/topartists.asp; Stand vom 22.11.2006. Zum Vergleich: Topseller in den USA sind die Beatles mit 169 Millionen Tonträgern, Led Zeppelin kommen auf 109,5, Aerosmith auf 65,5, Madonna auf 63, Metallica auf 57, Guns N' Roses auf 38,5 und Nirvana auf 25 Millionen. 


\section{Metal Blade Records - Heavy Metal in der Vorstadt}

Metallica waren in den frühen 1980er Jahren Teil der entstehenden Heavy Metal-Szene in der Greater L.A. Area. Sänger und Gitarrist James Hetfield war zu diesem Zeitpunkt einerseits bekennender Fan der Glam-Band Mötley Crüe und andererseits auf der Suche nach einer härteren Musik, als sie der US-Hard Rock der 1970er Jahre und in der Folge auch die Glam-, Hair Metalund Sleaze-Szene am Sunset Strip bieten konnten. Dieses Bedürfnis wurde von frühen Veröffentlichungen des britischen Heavy Metal und den Bands der so genannten New Wave of British Heavy Metal (NWOBHM) bedient, die aus dem Progressive Rock entliehene Songstrukturen mit der Energie und der Geschwindigkeit des Punk Rock kombinierten. Mit Judas Priest und Iron Maiden spielten zwei der einflussreichsten Bands des britischen Heavy Metal spätestens 1981 auch Konzerte in der Greater L.A. Area. ${ }^{6}$

Da solche Konzerte jedoch Ausnahmen waren, fanden sich die Anhänger des britischen Heavy Metal über Kontaktanzeigen in lokalen und überregionalen (Musik-)Zeitschriften. So lernten sich z.B. James Hetfield und Lars Ulrich, der spätere kreative und organisatorische Kern von Metallica, über eine Kontaktanzeige kennen. Eine wichtige Rolle kam dabei außerdem dem im Juni 1981 erstmals erschienenen britischen Magazin Kerrang zu, das auch in den USA rezipiert wurde. Ein weiterer Treffpunkt waren Plattenläden, die die gewünschte Musik im Repertoire hatten. Brian Slagel, der Gründer von Metal Blade Records, arbeitete Ende der 1970er Jahre in solch einem Plattenladen (Oz Records) und importierte Veröffentlichungen der NWOBHM nach Los Angeles. Slagel war zudem im so genannten Tape-Trading aktiv, das heißt, er tauschte Musikkassetten mit seiner Lieblingsmusik mit Brieffreunden aus aller Welt. Getauscht wurden neben inoffiziellen Konzertaufnahmen vor allem Demo-Tapes regionaler und überregionaler Bands. ${ }^{7}$ Ein

6 Judas Priest unternahmen bereits 1980 eine längere US-Tournee, deren genaue Konzerttermine mir nicht bekannt sind. Auf der nächsten US-Tournee, 1981, spielten Iron Maiden als Vorband von Judas Priest (vgl. http://www. rockdetector.com/officialbio,4773.sm; Stand vom 22.11.2006). Von Iron Maiden ist bekannt, dass sie im Rahmen ihrer »Killers World Tour am 5.6.1981 in El Paso und am 2.8.1981 in Long Beach auftraten (vgl. die Doppel-DVD The History of Iron Maiden. Part 1: The Early Days. EMI Music 724354431791 , 2004) - anzunehmen ist, dass sie diese Konzerte als Vorband von Judas Priest absolvierten.

7 Als Demo(nstration)-Tape bezeichnet man die meist ersten Studio-Aufnahmen einer Musikgruppe, mit denen diese versucht, einen Plattenvertrag zu ergattern. Demo-Tapes waren in der Prä-Internet-Zeit normalerweise nur auf Kon- 
Tape der oben genannten Iron Maiden z.B. erreichte Brian Slagel über einen Brieffreund aus Schweden bereits vor der Veröffentlichung des ersten Iron Maiden Albums 1980 durch EMI. Viele wichtige Protagonisten der internationalen Heavy Metal-Szene haben ihre Wurzeln in dieser Tape-TradingSzene (vgl. Mudrian 2004, Stratmann 2004). So auch Lars Ulrich, der spätere Schlagzeuger von Metallica, der nach seinem Umzug von Dänemark nach Newport Beach im Los Angeles County Ende der 1970er Jahre als europäischer Heavy Metal-Fan schnell ein guter Freund von Brian Slagel wurde. Der Heavy Metal-Netzwerkknoten in Los Angeles bestand damals laut Slagel aus genau drei Menschen: ihm selbst, Lars Ulrich und einem weiteren Freund: »Wir waren sogar bereit, zwei Stunden lang zu Plattengeschäften zu fahren, um die neueste Single zu finden« (Slagel, zit. n. Crocker 1993: 22).

Während man zum Sunset Strip aus den ganzen USA anreiste, um in den dortigen Klubs den individuellen Durchbruch zu schaffen, formierte sich die Heavy Metal-Szene in der Greater L.A. Area eher rund um einzelne Plattenläden und über Kontaktanzeigen. Der individualisierten Zusammenballung von Musikern und Musikerinnen an einem Ort, der Gegend um den Sunset Strip, stand hier ein mindestens über das Los Angeles County verteiltes suburbanes Netzwerk von Fans und Plattensammlern gegenüber, deren Gemeinsamkeit die Liebe zu britischem bzw. britisch beeinflusstem Heavy Metal war. So lebten z.B. die späteren Mitglieder von Metallica über die Greater L.A. Area verteilt in Newport Beach (Ulrich; L.A. County), Downey (Hetfield, McGovney; L.A. County) und Huntington Beach (Mustaine; Orange County). Über Brieffreundschaften mit Gleichgesinnten in der ganzen Welt im TapeTrading-Netzwerk war die Heavy Metal-Szene zudem von Beginn an international vernetzt und Brian Slagel entwickelte sich schnell zu einer zentralen Figur (vgl. German 2004). Er arbeitete in einem Plattenladen und als Radio-DJ, organisierte Konzerte und gab ab 1981 auch das Fanzine New Heavy Metal Review heraus. Da die Veröffentlichungen der NWOBHM oft bei kleinen unabhängigen Plattenfirmen erschienen, entstand 1981 bei Brian Slagel der Plan, ebenfalls eine eigene Plattenfirma zu gründen, um eine LP zu veröffentlichen, die die regionale Heavy Metal-Szene porträtieren sollte; aufgrund seiner vielfältigen Aktivitäten hatte er die Kontakte, um von Beginn an einen zumindest rudimentären Vertrieb für seine Plattenfirma gewährleisten zu können. 1982 erschien so Metal Massacre mit dem Untertitel The New Heavy Metal Review presents - die erste Veröffentlichung von Brian Slagels Metal Blade Records. Mit Steeler aus Tennessee war bereits mindestens eine überregionale Band vertreten. Metallica präsen-

zerten oder direkt bei der Band erhältlich, nichtsdestotrotz wurden manche dieser (Halb-)Veröffentlichungen vieltausendfach verkauft oder verschickt. 
tierten ihren ersten selbst geschriebenen Song »Hit The Lights« und eröffneten so die Reihe einflussreicher Heavy Metal-Bands, die auf einem Metal Massacre-Sampler von Metal Blade debütierten. ${ }^{8}$ Die Wurzeln im britischen Heavy Metal sind bei »Hit The Lights« deutlich zu hören. Dies gilt ebenfalls für die erste Veröffentlichung von Slayer, »Aggressive Perfector «, die 1983 auf Metal Massacre III erscheint. Die Intros beider Stücke sind von der 1979 veröffentlichten Live-Version des Judas Priest-Songs »Exciter « inspiriert.
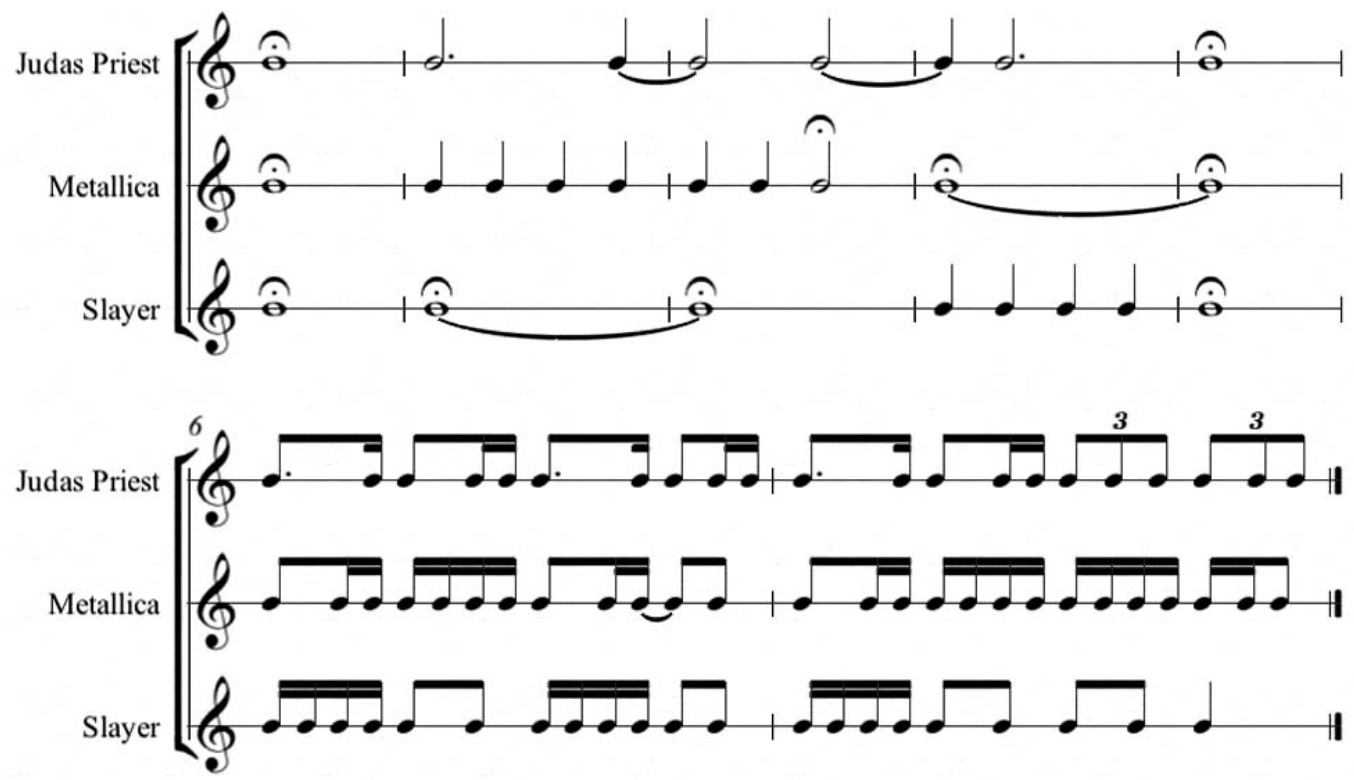

Abbildung 1: Vergleich des Anfangs von Judas Priest »Exciter «, Metallica »Hit The Lights« und Slayer »Aggressive Perfector «, letztere jeweils in der auf Metal Massacre erschienenen Version

Die Transkription bildet ausschließlich die Rhythmik der E-Gitarren ab. Charakteristisch ist bei allen drei Stücken der Übergang von den lang ausgehaltenen Noten bzw. Fermaten des Beginns und den schnellen Achteln und Sechzehnteln des folgenden Gitarrenriffs. Die Fermate zu Beginn des Notentextes wird bei Judas Priest und Metallica eingeblendet, bei Slayer startet sie mit dem Beginn des Stückes. Der Beginn des schnellen Gitarrenriffs wird von Judas Priest über Metallica zu Slayer immer weiter hinausgezögert eine Tatsache, die sich im Notentext an der Anzahl und Dauer der Fermaten erkennen lässt. Ausgedehnte Schlagzeugfills liegen unter den Fermaten, bzw. müssten bei Metallica und Slayer eigentlich noch als zusätzliche, an

8 Andere Bands sind Armored Saint (Metal Massacre II), Virgin Steele (Metal Massacre III), Trouble und Lizzy Borden (Metal Massacre IV), Fates Warning, Overkill, Metal Church und Voivod (Metal Massacre V), Possessed und Nasty Savage (Metal Massacre VI), Flotsam and Jetsam und Cryptic Slaughter (Metal Massacre VII) sowie Sacred Reich (Metal Massacre VIII). 
die Fermaten anschließende Takte eingefügt werden - bei Metallica und Slayer liegen unter der ersten Fermate sogar fast identische Schlagzeugfills. Während bei Judas Priest unter dem schnellen Gitarrenriff bereits ein durchgehender Schlagzeugbeat liegt, steht das jeweilige Riff bei Metallica und Slayer alleine.

Metallica zogen bereits ein paar Monate nach der Veröffentlichung von Metal Massacre von Los Angeles nach San Francisco. Ihre erste LP veröffentlichten sie 1983 unter anderem aufgrund von finanziellen Forderungen, die Metal Blade bzw. Brian Slagel nicht erfüllen konnten, bei Megaforce Records aus New Jersey. »Hit The Lights « blieb somit die einzige Metallica Veröffentlichung auf Metal Blade. Slayer wurden dagegen für die Jahre 1983-85 die kommerziell wichtigste Band des schnell wachsenden Labels. Ihre zweite und letzte LP für Metal Blade, Hell Awaits, verkaufte sich 1985 bereits im sechsstelligen Bereich. Die im Tape Trading bereits angelegte internationale Ausrichtung der Heavy Metal-Szene wurde von Metal Blade beständig weiter betrieben. Eine lokale oder regionale Ausrichtung war spätestens bei Metal Massacre $V$ von 1984, unter anderem mit Hellhammer aus der Schweiz, Voivod aus Kanada und Overkill aus New York, nicht mehr zu erkennen. Die Metal Massacre-Reihe wuchs bis 1988 auf neun Folgen an und bildete den Grundstein für ein Label, das mittlerweile Büros in den USA, der BRD und Japan unterhält und bis heute eine der wichtigsten unabhängigen auf Heavy Metal spezialisierten Plattenfirmen ist - eine Geschichte, die auf einer (Einzel-)Initiative innerhalb eines sich herausbildenden internationalen Netzwerks von Heavy Metal-Fans und -Aktivisten beruht.

\section{SST Records - Hardcore (und mehr) in den strandnahen Vorstädten}

Im Gegensatz zum Heavy Metal und der Szene am Sunset Strip erscheint Hardcore in der Greater L.A. Area als lokal begrenzteres Phänomen. Es entstand ebenfalls Ende der 1970er, Anfang der 1980er Jahre in den strandnahen Vorstädten und verbreitete sich von dort aus weiter. Hardcore war wie Heavy Metal eine suburbane Szene, die sich von einer als innerstädtisch verstandenen Gegend, wie sie der Sunset Strip verkörpert, abgrenzte. Jedoch scheinen hier persönliche Kontakte an der Schule, in der unmittelbaren Nachbarschaft oder bei Freizeitaktivitäten wie Skaten oder Surfen erheblich wichtiger gewesen zu sein als die Kontaktanzeigen der Heavy Metal-Szene. 
Mark Spitz und Brendan Mullen (2001) beschreiben das Dasein als Punk in den vorstädtischen Gebieten als eine Situation, in der man permanent latenter und expliziter körperlicher Gewalt ausgesetzt war:

»Eugene: >[...] there were a lot of kids from the beaches who were getting seriously fucked up by these long haired redneck hicks in their $4 \times 4$ vehicles, real Lynyrd Skynyrd kind of guys [...]. No disrespect against the Hollywood party punks who'd been around longer, but they just weren't prepared to defend us« (Spitz/Mullen 2001: 192).

Solche vierradgetriebenen Fahrzeuge waren ein Statussymbol in der damaligen Surfer-Szene, die wie die damalige Skater-Szene Hard Rock als Musikstil bevorzugte.

Der Konformitätsdruck erscheint in den Vorstädten erheblich stärker ausgeprägt gewesen zu sein als am Sunset Strip, an dem visuelle Extravaganz viel stärker zum Alltagsbild gehörte. Die langen Haare der männlichen Heavy Metal-Fans in den Vorstädten entsprachen dagegen anscheinend eher dem tolerierten Aussehen. Dies bedingte für Punks eine zumindest gefühlte Notwendigkeit der Verteidigung gegen gewalttätige Angriffe, die wiederum zu einer höheren Gewaltbereitschaft der vorstädtischen Punk- bzw. der entstehenden Hardcore-Szene führte. Diese stärkere Gewaltbereitschaft brach auch bei Tumulten vor, während oder nach Konzerten aus, die wiederum die Punks vom Sunset Strip abschreckten, da Gewalt hier »spielerischer « verstanden wurde (vgl. ebd.: 217-225). Da Hardcore so ein potentiell gewalttätiges Image bekam, wurde die Szene im Umkehrschluss wiederum zu einem Anziehungspunkt für gewaltbereite Jugendliche, die mehr an den Schlägereien als an der Musik interessiert waren:

»Stan Ridgway: >Black Flag would play and take no responsibility for what was going on in the crowd. [...] Pogoing became slamming. Beating each other up.... and loving it. [...] So I headed way north of it, too « (Spitz/Mullen 2001: 224).

Gewalt erschien als eine der Hauptbegründungen der Trennung in eine innerstädtische Punk- und vorstädtische Hardcore-Szene. Die bereits 1976 als Panic gegründete und 1978 umbenannte Band Black Flag aus Redondo Beach im Los Angeles County prägte diese Entwicklung entscheidend mit. Die u.a. aus der Gewaltproblematik resultierenden Schwierigkeiten, ihre Musik öffentlich zu präsentieren, führten bei Bandgründer und -leader Greg Ginn zu der Idee einer kompletten Selbstorganisation der eigenen Karriere - in Abgrenzung sowohl zum etablierten Musikbusiness als auch zu den bestehenden unabhängigen Strukturen. 
Black Flag und Greg Ginn organisierten ihre Konzerte und Tourneen selbst, druckten und verteilten Werbematerialien und gründeten 1978 auch ihre eigene Plattenfirma SST Records als Erweiterung der gleichnamigen Elektronikfirma des ehemaligen Ökonomiestudenten Greg Ginn. Die erste Veröffentlichung von SST war natürlich eine Aufnahme von Black Flag, die EP Nervous Breakdown.

SST wurde im Gegensatz zu Metal Blade nicht von Fans, sondern von den Musikern selbst betrieben. Überregionale Kontakte entstanden nicht über ein Tape Trading-Netzwerk wie im Fall der Heavy Metal-Szene, sondern wurden durch selbst organisierte Tourneen zu anderen lokal ausgerichteten Szenen in den USA aufgebaut. Eine solche Szene war z.B. die um das Dischord Label in Washington D.C. zentrierte Hardcore-Szene. Das so entstandene Netzwerk war viel mehr an Auftrittsorte wie Jugendzentren gekoppelt als das Tape-Trading-Netzwerk, das oft von Privatwohnungen aus betrieben wurde. Auch Mitmusiker wurden in der Hardcore-Szene viel stärker über persönliche Kontakte gesucht als über Anzeigen. Henry Rollins z.B., von 1981 bis 1986 Sänger von Black Flag, stammte zwar aus Washington D.C., war aber bereits Fan der Band und wurde während einer laufenden Tournee in Washington rekrutiert. Dagegen waren sowohl im Heavy Metal als auch in der Szene am Sunset Strip die handwerklichen musikalischen Fähigkeiten ein viel entscheidenderes Kriterium als persönliche Bekanntschaft bei der Anwerbung neuer Mitglieder.

Über Black Flag war SST zwar musikalisch mit der Hardcore-Szene assoziiert, in der Praxis wurde Hardcore bei SST jedoch weniger als Musikstil denn als eine Haltung der Selbstorganisation begriffen. Veröffentlicht wurde deshalb von Beginn an ein erheblich breiteres Spektrum an Musikstilen, bzw. die Projekte einer Gruppe assoziierter Musiker, die sich vom Punk und Hardcore kommend in unterschiedliche stilistische Richtungen weiterentwickelten. Das beste Beispiel hierfür sind Black Flag selbst, die auf späteren Veröffentlichungen (1984-1986) schleppenden, von Black Sabbath inspirierten Hard Rock und auch Jazz Rock Elemente integrierten. Minutemen aus San Pedro im Los Angeles County - die zweite Band, die auf SST veröffentlichte - integrierten Latin Elemente und immer mehr Rock in ihre Musik. Immer wieder bildeten sich auch neue Gruppen aus anderen auf SST veröffentlichenden Bands. Unterstützung erfuhren SST, Black Flag und andere regionale Hardcore-Bands von Beginn an vom einflussreichen Radio DJ Rodney Bingenheimer, der bereits in der Glam-Szene der 1970er Jahre am Sunset Strip eine wichtige Rolle gespielt hatte (vgl. Hickenlooper 2003). In der Folge wurde SST zu einer der wichtigsten und auch erfolgreichen unabhängigen US-amerikanischen Plattenfirmen für Rock und Hardcore der 1980er 
Jahre. Zwischen 1978 und 1984 wurden ungefähr siebzig Veröffentlichungen realisiert, zwischen 1985 und 1987 erschienen mindestens 130 weitere Veröffentlichungen auf SST.

Ende der 1980er Jahre begann der Stern von SST aus mehreren Gründen zu sinken. Zum einen setzten interne Querelen um Geld ein, die den guten Ruf der Plattenfirma beschädigten (vgl. Lang 1998) und zum anderen waren Greg Ginns Nachfolgeprojekte der 1986 aufgelösten Black Flag kommerziell nicht so erfolgreich. Außerdem bekamen in der Folge des medialen Hypes um Nirvana und die Grunge-Bewegung viele Bands plötzlich Angebote der internationalen Schallplattenindustrie. Firehose, die Nachfolgeband von Minutemen, wechselten z.B. zu Columbia, Hüsker Dü, eine der meistverkaufenden Bands von SST, zu Warner Brothers und Sonic Youth zu Geffen. Mit Soundgarden hatte man auch eine Band aus der Grunge-Stadt Seattle im Programm, die jedoch zu A\&M Records wechselte und dort dann Millionen von Platten verkaufte. SST existiert bis heute, verwaltet jedoch hauptsächlich das Archiv der Veröffentlichungen aus den 1980er Jahren. Neuveröffentlichungen sind, wenn überhaupt, Projekte von Gründer und Eigentümer Greg Ginn.

\section{Outro}

Als mögliche Modelle der Organisation regionaler, um Stile populärer Musik zentrierter Szenen identifiziert werden können erstens die regionale Selbstorganisation von Musikern, zweitens die regionale Selbstorganisation international vernetzter Fans und drittens die regionale Zusammenballung von Musikern, die hoffen, an der internationalen Unterhaltungsindustrie teilzuhaben. Über die Unterschiede, aber vor allem auch die Gemeinsamkeiten dieser drei modellhaften Szenen ließe sich noch viel mehr berichten. Vernachlässigt wurde zum Beispiel die Rolle von Ozzy Osbourne, der für alle drei hier beschriebenen Szenen wichtig war. Seine frühere Band Black Sabbath war eine wichtige Inspirationsquelle für Black Flag und SST. Im Vorprogramm seiner 1981er-Tournee spielten die britischen Motörhead, eine bei Punks und Heavy Metal-Fans gleichermaßen beliebte Band, erstmals in der Greater L.A. Area. Zu seiner Band in diesem Jahr gehörte mit Randy Rhoads ein stilistisch äußerst einflussreicher und virtuoser Leadgitarrist, der vor seinem Engagement bei Ozzy Osbourne in der Glam Metal-Band Quiet Riot spielte. Sein späterer Bassist Robert Trujillo stammte zum einen aus der ehemaligen Hardcore-Band Suicidal Tendencies und ist zum anderen mittlerweile Bassist bei Metallica. Dementsprechend existieren die darge- 
stellten Szenen in der Realität auch beileibe nicht so getrennt voneinander, wie hier suggeriert. Gerade die professionellen Aktivisten aller drei Szenen kennen sich oft persönlich, da man sich in geschäftlichen Angelegenheiten begegnet oder zumindest nahe gekommen ist.

Die beschriebenen Unterschiede, wie die größere Vereinzelung in der Szene am Sunset Strip und dementsprechend ein größeres Gemeinschaftsgefühl in den anderen beiden Szenen, sind vor allem als Tendenzen zu verstehen. Gleiches gilt für das größere Augenmerk, das am Sunset Strip und im Heavy Metal auf die musikalische Kompetenz potentieller Mitmusiker gelegt wurde.

Metal Blade als Beispiel für die Selbstorganisation von Fans und SST als Beispiel für die Selbstorganisation von Musikern sind als lokale Organisationsformen eigentlich überall auf der Welt möglich, die ständig und überall geschehen - mit jeweils wechselndem Erfolg und Einfluss auf die überregionale Wahrnehmung von Szenen und Stilistiken populärer Musik. Sie sind immer eine der Triebfedern für die stilistische und inhaltliche Differenzierung populärer Musik.

Kommerzieller Erfolg ist bei allen drei Organisationsmöglichkeiten zumindest temporär möglich. Musikalische Innovationen entstanden eher in den beiden Fällen, in denen an dem als kleinsten gemeinsamen musikalischen Nenner zu definierenden Mainstream vorbei musiziert wurde. Am Sunset Strip ging es stattdessen um einen Zugang zu eben diesem Mainstream, der sich über die Inkorporation neuer Musikergenerationen und ihrer Ideen langsam aber beständig modernisierte.

\section{Literatur}

Christie, Ian (2003). Sound of the Beast. The Complete Headbanging History of Heavy Metal. New York: Harper Collins.

Crocker, Chris (1993). Metallica. Nothing Else Matters. St Andrä-Wördern: Hannibal.

Davis, Mike (1994). City of Quartz. Ausgrabungen der Zukunft in Los Angeles. Berlin, Göttingen: Schwarze Risse/Rote Straße.

German, Eric (2004). »Behind the Screams Part 2: Metal Blade Records. « In: http: // www. metalupdate.com/interviewmetalblade.html (Stand vom 22.11.2006).

Lang, Dave (1998). »The SST Records story - Part 3. «In: http: //www.furious.com/ perfect/sst3.html (Stand vom 22.11.2006).

Moore, Allan F. (2001). Rock: The Primary Text. Aldershot u.a.: Ashgate (2. Aufl.).

Mudrian, Albert (2004). Choosing Death. The Improbable History of Death Metal and Grindcore. Los Angeles: Feral House.

Slobin, Mark (2000). Subcultural Sounds. Micromusics of the West. Hanover, London: Wesleyan University Press (2. Aufl.). 
Spitz, Marc / Mullen, Brendan (2001). We Got the Neutron Bomb. The Untold Story of L.A. Punk. New York: Three Rivers Press.

Stratmann, Holger (Hg.) (2004). Rock Hard Mania. 20 Jahre Rock und Metal im Überblick. Dortmund: Rock Hard.

Walser, Robert (1993). Running With The Devil. Power, Gender and Madness in Heavy Metal Music. Hanover, N.H., London: Wesleyan University Press.

\title{
Diskographie
}

Aerosmith (1976). Rocks. CBS, 32360.

Black Flag (1978). Nervous Breakdown. SST, 001.

Guns N' Roses (1987). Appetite for Destruction. Geffen, 424 148-1.

Judas Priest(1979/2001). »Exciter."Auf: Unleashed In The East. CBS, 50212.

Metallica (1982). »Hit The Lights. « Auf: V.A. - Metal Massacre 1. Metal Blade, MBR 1001.

Quiet Riot (1983). »Cum On Feel The Noize.« Auf: Metal Health. Epic, EPC 25322.

Slade (1973). »Cum On Feel The Noize.« Auf: Sladest. Polydor, 2442119.

Slayer (1983). »Aggressive Perfector.« Auf: V.A. - Metal Massacre 3. Metal Blade, MBR 1008.

Slayer (1985). Hell Awaits. Metal Blade Records, 72297-1.

Van Halen (1977). »Eruption. « Auf: Van Halen. Warner Brothers, WB 56470.

Various Artists (1982-1986). Metal Massacre I-VII. Metal Blade, MBR 1001/1004/ 1008/1012/1021/1036/1054.

Various Artists (1986/1988). Metal Massacre VIII/IX. Metal Blade, 72178-1/72246-1.

\section{Filme}

Dunn, Sam / McFayden, Scott (2006). Metal. A Headbanger's Journey. München: Constantin (DVD).

Hickenlooper, George (2003). Mayor of the Sunset Strip. London: Tartan Video (DVD).

Spheeris, Penelope (1988). The Decline of the Western Civilisation Part 2: The Metal Years. O.O.: I.R.S. World Media (Video).

\begin{abstract}
Regional scenes of popular music can be regarded as driving forces of popular music history. As examples for typical structural features and for the development of regional scenes three such scenes from the Greater Los Angeles Area are compared, which arose more or less at the same time: the Hair Metal scene around Sunset Strip, the metal scene, which had its centre at Metal Blade Records, and the Hardcore scene of SST Records. The identified forms of organization range from a conglomerate of musicians, keen on a major label deal, to a label founded by fans and a label founded by musicians.
\end{abstract}

